\title{
Thermodynamic consistency of the equation of state of strongly interacting matter
}

\author{
J. Randrup ${ }^{a}$ and P.V. Ruuskanen ${ }^{b}$ \\ ${ }^{a}$ Nuclear Science Division, Lawrence Berkeley National Laboratory, Berkeley, California 94720, USA and \\ ${ }^{b}$ Department of Physics, University of Jyväskylä, Finland
}

(Dated: December 12, 2003)

\begin{abstract}
Addressing strongly interacting matter in the region of energy density where the hadronic gas phase coexists with the quark-gluon plasma phase, we discuss how thermodynamic consistency can be used to constrain the equation of state for uniform matter and we illustrate the method by constructing a $T_{c}$-dependent family of thermodynamically consistent equations of state based on simple spline interpolations between the gas and plasma phases.

PACS numbers: 25.75.-q, 05.70.-a, 64.70.-p, 64.90.+b
\end{abstract}

Strongly interacting matter is expected to exhibit a first-order phase transition from a hadronic gas to a quark-gluon plasma with an associated critical temperature $T_{c} \approx 150-200 \mathrm{MeV}$. The exploration of this fundamental phenomenon has long been the focal point of relativistic heavy-ion physics.

The identification of useful experimental signals is dependent on our ability to develop suitably realistic models for the dynamical evolution of the system. Microscopic transport treatments are hampered by the fact that the relevant degrees of freedom differ in the two phases. This basic problem is absent in fluid dynamics, which is inherently macroscopic, and such a framework is therefore well suited for propagating the system as it expands through the region of phase coexistence where the microscopic structure of the system changes. The fluid-dynamical framework has the additional advantage that its key physics ingredient, the equation of state $p(\varepsilon)$, is precisely the central object of inquiry. However, the calculation of the equation of state of strongly interacting matter in the region of phase-coexistence presents a daunting challenge and it is necessary to employ approximate forms when studying this important part of the phase diagram.

When a system has a first-order phase transition, then its thermodynamic potential presents a convex anomaly and the corresponding thermodynamic quantities, such as temperature and pressure, have a non-monotonic dependence on the energy density [1]. The presence of a local convexity implies the existence of two separate phase points that have a common tangent and which may therefore coexist in thermodynamic equilibrium, with the resulting (average) energy density being determined by the relative proportion of the two phases. Thus, throughout this region of phase coexistence, systems prepared in spatially uniform configurations are thermodynamically unstable since they can be rearranged into more favorable configurations by phase separation. The region of coexistence naturally divides into three sectors: the two regions near the boundaries where the thermodynamic potential is still locally concave and the central sector where it is locally convex. In the concave sectors, a spatially uniform configuration is metastable and phase separation occurs by nucleation since a finite disturbance is required to trig- ger the separation. By contrast, in the central sector where the concavity is present, even infinitesimal deviations from uniformity will become amplified and phase separation will thus occur spontaneously. Under such circumstances, uniform matter is mechanically unstable and will exhibit spinodal decomposition, the end results of which is the spatial separation of the initially uniform system into a macroscopically non-uniform configuration with the hadron and plasma phases coexisting in thermodynamic equilibrium.

Traditionally, fluid-dynamical treatments have suppressed these instabilities by replacing the pressure function $p(\varepsilon)$ through the unstable region by the constant value $p_{c}$ associated with a phase mixture having the same average energy density (see Refs. [2-4], for example). However, since a spatially separated phase mixture cannot develop instantly, due to the energy transport required, it is necessary to investigate the dynamics of the associated decomposition process. This issue was addressed recently in a study that employed a cubic spline function for the equation of state in the coexistence region and then obtained simple expressions for the resulting spinodal growth rates [5]. That simple treatment suggested that the degree of amplification achieved during the traversal of the spinodal phase region may be sufficient to make the ensuing spinodal pattern useful as a diagnostic tool for probing the hadronization process.

The present Brief Report focusses on thermodynamic consistency in the construction of the equation of state of strongly interacting uniform matter in the phasecoexistence region, a property that is generally not present in a simple spline approximation.

We consider first thermal equilibrium of a spatially uniform single-phase system in the thermodynamic limit. Since the present note merely serves to illustrate how thermodynamic consistency may be incorporated, it is preferable to consider the simplest scenario. The chemical potentials are therefore taken to be zero. However, it is expected that the first-order phase transition occurs only when the baryon chemical potential exceeds a certain critical value, so it is important to adapt the method appropriately to any specific scenario under study. While this critical value must ultimately be determined experimentally, very recent lattice calculations suggest that it 
is in fact relatively small [6]. If this is indeed the case, our present results would then hold approximately for the midrapidity region at RHIC.

In the thermodynamic limit, the energy $E$, the entropy $S$, and the free energy $F=E-T S$ are all proportional to the volume $V$,

$$
\begin{aligned}
E(V, T) & =V \varepsilon(T), \\
S(V, T) & =V \sigma(T), \\
F(V, T) & =V f(T),
\end{aligned}
$$

and, with the chemical potentials fixed, the reduced quantities $\varepsilon, \sigma$, and $f=\varepsilon-T \sigma$ depend only on the temperature $T$. The pressure and the entropy are given as partial derivatives of $F$,

$$
\begin{aligned}
p(T) & =-\left(\frac{\partial F}{\partial V}\right)_{T}=-f(T), \\
S(V, T) & =-\left(\frac{\partial F}{\partial T}\right)_{V}=-V \frac{d f}{d T} .
\end{aligned}
$$

We thus have $\sigma=-d f / d T=d p / d T$. (This result also follows from the Maxwell relations of thermodynamics which express the mathematical fact that the mixed derivaties of the thermodynamic potential are identical, $\partial^{2} F / \partial T \partial V=\partial^{2} F / \partial V \partial T$, i.e. $\partial p / \partial T=\partial S / \partial V=\sigma$.) This relation implies $d(T \sigma)=d(\varepsilon+p)$. Furthermore, since Eq. (4) implies $d p / d T=\sigma+T d \sigma / d T-d \varepsilon / d T$, we also have $T d \sigma / d T=d \varepsilon / d T$. Hence the following relations hold generally,

$$
\begin{aligned}
& d p=\sigma d T, \\
& d \varepsilon=T d \sigma .
\end{aligned}
$$

We refer to these as the Maxwell relations.

As discussed above, it is possible for two such thermodynamic phases to coexist when the thermodynamic potential for the uniform system has a convex anomaly. The two coexisting phase points are then those that define the common tangent. In addition to having the same temperature $T_{c}$, the existence of a common tangent implies that the two phases also have the same pressure $p_{c}$ (since $\left.(\partial F / \partial V)_{T}=-p\right)$.

We now consider this type of situation and address the form of the thermodynamic functions through the intermediate region of energy density where a uniform configuration is mechanically unstable (or at most metastable) against separation into the two coexisting thermodynamic phases. Although our considerations are general, we have in mind specifically the coexistence of a hadronic gas phase (of energy density $\varepsilon_{H}$ ) and a quarkgluon plasma (of energy density $\varepsilon_{Q}$ ).

From the first Maxwell relation $d p=\sigma d T$ (6) we find

$$
\frac{d p}{d \varepsilon} d \varepsilon=d p=\sigma d T=[\varepsilon+p] \frac{d T}{T} .
$$

Thus, if the pressure is known as a function of energy density, $p(\varepsilon)$, then $T(\varepsilon)$ can be determined,

$$
T\left(\varepsilon_{2}\right)=T\left(\varepsilon_{1}\right) \exp \left[\int_{\varepsilon_{1}}^{\varepsilon_{2}} \frac{d p(\varepsilon)}{d \varepsilon} \frac{d \varepsilon}{\varepsilon+p(\varepsilon)}\right],
$$

where $\varepsilon_{1}$ and $\varepsilon_{2}$ are two arbitrary energy densities. This relation holds generally and it can be utilized to constrain the equation of state whether or not there is a phase transition. When a first-order phase transition is present, $T(\varepsilon)$ should be equal to $T_{c}$ at the coexistence boundaries, $\varepsilon_{H}$ and $\varepsilon_{Q}$, and we obtain an integral condition,

$$
\int_{\varepsilon_{H}}^{\varepsilon_{Q}} \frac{d p(\varepsilon)}{d \varepsilon} \frac{d \varepsilon}{\varepsilon+p(\varepsilon)}=0
$$

which must be obeyed by any thermodynamically viable pressure function $p(\varepsilon)$.

From the second Maxwell relation (7), $\beta d \varepsilon=d \sigma$, we obtain directly a condition on $\beta(\varepsilon)=1 / T(\varepsilon)$,

$$
\int_{\varepsilon_{1}}^{\varepsilon_{2}} \beta(\varepsilon) d \varepsilon=\int_{\sigma_{1}}^{\sigma_{2}} d \sigma=\sigma_{2}-\sigma_{1} .
$$

The general relations (9-11) may be particularly useful if the equation of state is known at the energy densities $\varepsilon_{1}$ and $\varepsilon_{2}$, (such as may be the case in the limits $\varepsilon_{1} \rightarrow 0$ and $\left.\varepsilon_{2} \rightarrow \infty\right)$. For the first-order phase transition, we may use the fact that $\sigma_{Q}-\sigma_{H}=\beta_{c}\left(\varepsilon_{Q}-\varepsilon_{H}\right)$ to recast (11) as a Maxwell-type condition,

$$
\int_{\varepsilon_{H}}^{\varepsilon_{Q}}\left[\beta(\varepsilon)-\beta_{c}\right] d \varepsilon=0
$$

which requires the vanishing of the net area between the actual curve $\beta(\varepsilon)$ and the (constant) value $\beta_{c}=1 / T_{c}$ pertaining to the phase mixture.

There is of course no gurantee that the thermodynamic consistency conditions (10) and (12) will be satisfied by a given function $p(\varepsilon)$, but they either succeed or fail together: If one of those integral conditions is met, then the other one is also met, since the basic relation $d(T \sigma)=d(\varepsilon+p)$ guarantees that $T d \sigma=d \varepsilon$ implies $\sigma d T=d p$ and vice versa. Once $T(\varepsilon)$ is known, the entropy then readily follows, $\sigma(\varepsilon)=(\varepsilon+p(\varepsilon)) / T(\varepsilon)$.

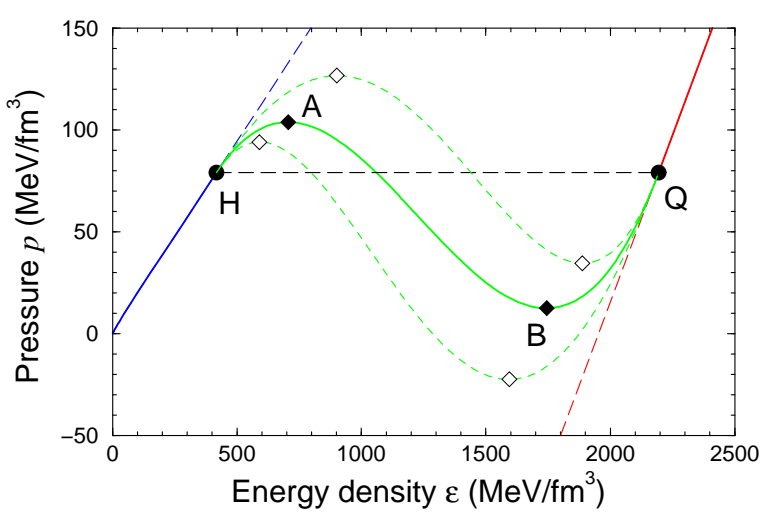

FIG. 1: The equation of state obtained by interpolating between the hadronic gas and the quark-gluon plasma by means of a cubic spline function (solid). Also shown are the equations of state obtained by augmenting the spline (13) with a quartic adjustment (14) using $p_{0}= \pm 50 \mathrm{MeV} / \mathrm{fm}^{3}$ (dashed). 
After the above general considerations, we now illustrate how a thermodynamically consistent equation of state may be obtained by a spline procedure.

In order to obtain a quantitative result, we adopt specific forms for the equation of state in the mechanically stable gas and plasma regions. As is commonly done [2-5], we describe the hadronic phase as an ideal gas of hadrons, including 14 known species $\{\pi, K, \eta, \rho, \ldots, N, \Lambda, \Sigma, \Delta\}$, while the plasma is an ideal gas of of gluons and $u, d, s$ quarks and antiquarks (with vanishing chemical potentials). The specification of the critical temperature (for which we take $T_{c}=170 \mathrm{MeV}$ ) determines the associated bag constant. The corresponding critical pressure is then $p_{c} \approx 80 \mathrm{MeV} / \mathrm{fm}^{3}$ and the energy densities of the two coexisting phases are $\varepsilon_{H} \approx 400 \mathrm{MeV} / \mathrm{fm}^{3}$ and $\varepsilon_{Q} \approx 2200 \mathrm{MeV} / \mathrm{fm}^{3}$, implying a latent heat of $\Delta \varepsilon \approx 1800 \mathrm{MeV} / \mathrm{fm}^{3}$. Furthermore, $v_{H}^{2}=(d p / d \varepsilon)_{Q} \approx 0.20$ and $v_{Q}^{2}=(d p / d \varepsilon)_{Q}=\frac{1}{3}$ are the squares of the sound speeds in the coexisting systems.

From this quite conventional starting point, we first follow the procedure employed in Ref. [5] and consider the cubic spline function $\tilde{p}(\varepsilon)$ that matches the pressure and its slopes at $\varepsilon_{H}$ and $\varepsilon_{Q}$,

$$
\tilde{p}(\varepsilon)=p_{c}+\xi(\varepsilon)\left[\left(\varepsilon_{Q}-\varepsilon\right)^{2} v_{H}^{2}-\left(\varepsilon-\varepsilon_{H}\right)^{2} v_{Q}^{2}\right] \bar{\xi}(\varepsilon),
$$

where the interpolation parameter is $\xi \equiv\left(\varepsilon-\varepsilon_{H}\right) / \Delta \varepsilon$ and $\bar{\xi} \equiv 1-\xi$. The resulting pressure function is displayed in Fig. 1. While having a smooth dependence on $\varepsilon$ everywhere, it generally does not conform exactly with the conditions of thermodynamic consistency.

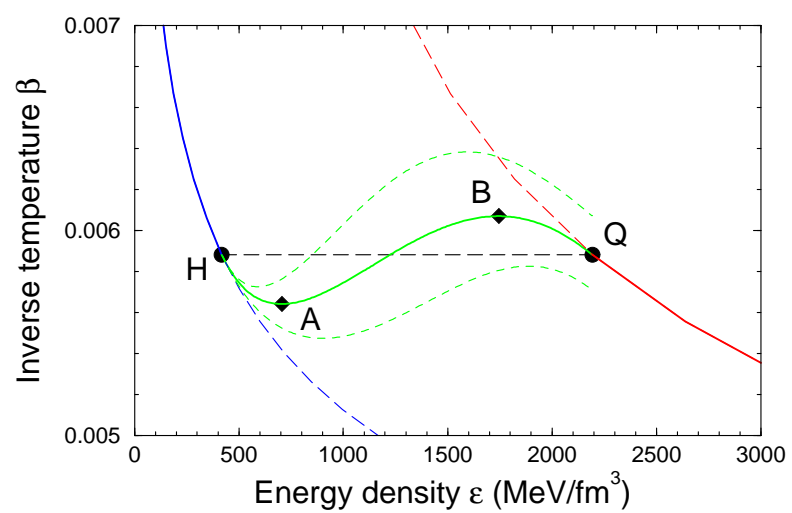

FIG. 2: The inverse temperature $\beta(\varepsilon)$ as obtained by applying the thermodynamic integral relation (9) to the functions $p(\varepsilon)$ shown in Fig. 1, starting from $\varepsilon_{H}$ and ending at $\varepsilon_{Q}$.

In order to determine to what degree the spline approximation (13) violates the Maxwell condition (12), we calculate $\beta(\varepsilon)$ by using Eq. (9). The resulting function is shown in Fig. 2. The Maxwell condition (12) demands the vanishing of the net area between the curve $\beta(\varepsilon)$ (solid) and the constant value $\beta_{c}$ associated with the mixed phase (dashed), which is ensured if $\beta(\varepsilon)$ returns to the critical value $\beta_{c}$ at the boundary $\varepsilon_{Q}$. As is apparent from the figure, the condition happens to be well satisfied for the particular case considered.

A wider range of candidate equations of state can be explored by augmenting the above cubic spline (13) and thus consider $p(\varepsilon)=\tilde{p}(\varepsilon)+\delta p(\varepsilon)$. In order to maintain the match of pressure and speed of sound at the coexistence points the value and slope of $\delta p(\varepsilon)$ must vanish at $\varepsilon_{H}$ and $\varepsilon_{Q}$. We therefore employ a quartic term,

$$
\delta p(\varepsilon)=16 p_{0} \xi(\varepsilon)^{2} \bar{\xi}(\varepsilon)^{2} .
$$

This term is peaked at the midpoint $\frac{1}{2}\left(\varepsilon_{H}+\varepsilon_{Q}\right)$ where $\xi=\frac{1}{2}$ and where it has the value $p_{0}$. Its main effect is to shift the inflection point of $p(\varepsilon)$ in the direction of the sign of the strength parameter $p_{0}$. Figures 1-2 include the results of using the values $p_{0}= \pm 50 \mathrm{MeV} / \mathrm{fm}^{3}$. It is evident from Fig. 2 that these values of $p_{0}$ lead to a significant degree of thermodynamic mismatch at $\varepsilon_{Q}$.

By adjusting $p_{0}$ it is possible to obtain exact thermodynamic consistency. We have determined the corresponding optimal value to be $p_{0} \approx-0.8 \mathrm{MeV} / \mathrm{fm}^{3}$ which is hardly significant as the corresponding changes in $p(\varepsilon)$ and $T(\varepsilon)$ are invisible on the plots.

To further broaden the range of scenarios considered, we now vary the specified critical temperature $T_{c}$ and consider also the values $160 \mathrm{MeV}$ and $180 \mathrm{MeV}$. The corresponding spline approximations to the equation of state are shown in Fig. 3, while Fig. 4 displays the associated temperature functions resulting from the thermodynamic relation Eq. (9). A quick glance at Fig. 4 suggests that the spline approximation leads to a good degree of thermodynamic consistency also for these cases. However, a closer inspection reveals that the the two curves have somewhat larger discontinuities of $T(\varepsilon)$ at $\varepsilon_{Q}$ (these are still quite small and difficult to spot on the plot). If the parameter $p_{0}$ is adjusted to ensure a perfect match, the required values are $p_{0} \approx-3.6,2.9 \mathrm{MeV} / \mathrm{fm}^{3}$ for $T_{c}=160,180 \mathrm{MeV}$, respectively.

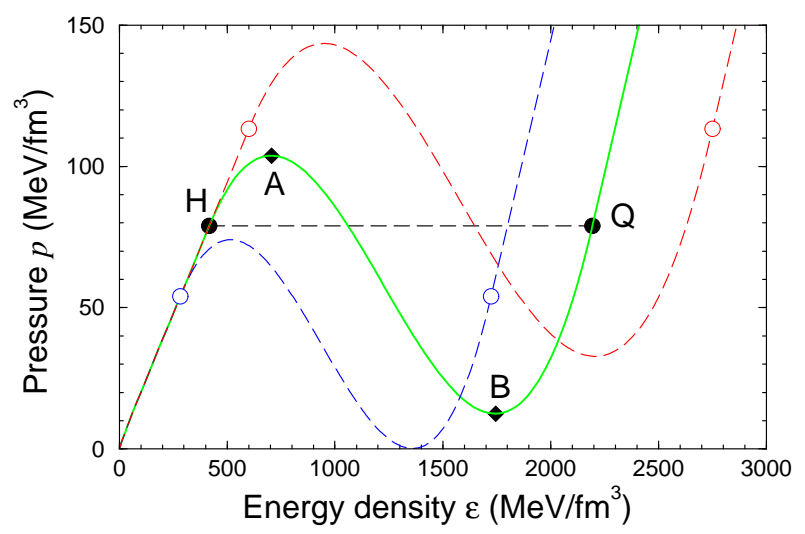

FIG. 3: The equation of state $p(\varepsilon)$ obtained with the cubic spline approximation (13) for three values of the critical temperature, $T_{c}=160,170,180 \mathrm{MeV}$. The coexistence points are indicated and, for $T_{c}=170 \mathrm{MeV}$, the constant-pressure Maxwell contruction is indicated. 


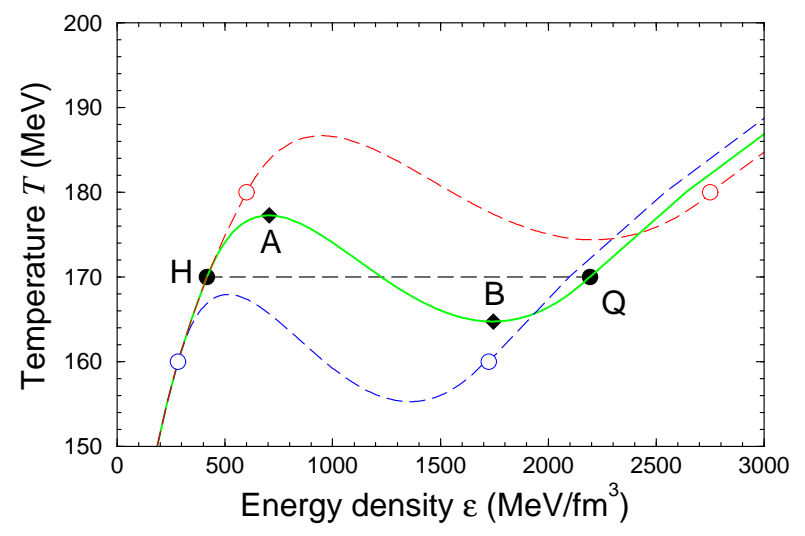

FIG. 4: The dependence of the temperature $T$ on energy density $\varepsilon$ as obtained by using Eq. (9) with the the three equations of state $p(\varepsilon)$ shown in Fig. 3, The phase-coexistence points are indicated (All three curves have slight discontinuities at $\varepsilon_{Q}$ that are barely visible to the eye).

It follows that there exists a particular value of $T_{c}$ for which the unadjusted cubic spline function satisfies the thermodynamic consistency condition exactly. In the present treatment, this special value comes out as $T_{c} \approx 172 \mathrm{MeV}$. (It should be recognized that the precise numbers obtained for the parametrized forms depend on the specific physics input, particularly the specification of the hadron species and their masses. Consequently, any application of the method proposed here should of course refit these values appropriately. Still, we find it worthwhile to quote the precise numbers so that readers with special interest in the topic may verify the results.)

In summary, we have shown how a thermodynamically consistent equation of state for uniform matter must generally satisfy a Maxwell-type integral condition, which thus provides a constraint on the equation of state for uniform matter prepared in the phase-coexistence region. This may constitue a useful aid for the development of approximate equations of state for the phase-coexistence region. Since the analysis is quite general, the proposed method of approximation may be useful also outside the area of strong-interaction physics on which we have focussed here. Of course, as already pointed out, the procedure needs to be carried out for the applicable values of the chemical potentials.

In order to provide quantitative results, we have employed schematic representations of the two single-phase regimes as ideal gases of either elementary hadrons or quarks and gluons, as is common practise. Examining a family of candidate pressure functions $p(\varepsilon)$ constructed by making a quartic adjustment of the cubic spline function that smoothly connects the two single-phase regions, we have found that the simple (unadjusted) spline form in fact satisfies the thermodynamic consistency requirement quite well for a broad range of critical temperatures. Thus this simple approximation may be useful for dynamical studies involving the phase-coexistence region, such as fluid-dynamical calculations of the rapidly expanding system formed in a high-energy nuclear collision.

This work was supported by the Office of Energy Research, Office of High Energy and Nuclear Physics, Nuclear Physics Division of the U.S. Department of Energy under Contract No. DE-AC03-76SF00098.
[1] See, for example, P.M. Chaikin and T.C. Lubensky, Principles of Condensed Matter Physics, Cambridge University Press (1995).

[2] D.H. Rischke, Y. Pürsün, and J.A. Maruhn, Nucl. Phys. A595, 383 (1995).

[3] J. Sollfrank, P. Huovinen, M. Kataja, P.V. Ruuskanen, M. Prakash, and R. Venugopalan, Phys. Rev. C 55, 392
(1997).

[4] T. Hirano and K. Tsuda, Phys. Rev. C 66, 054905 (2002).

[5] J. Randrup, nucl-th/0308271.

[6] F. Karsch, C.R. Allton, S. Ejiri, S.J. Hands, O. Kaczmarek, E. Laermann, and C. Schmidt, hep-lat/0309116 (2003). 


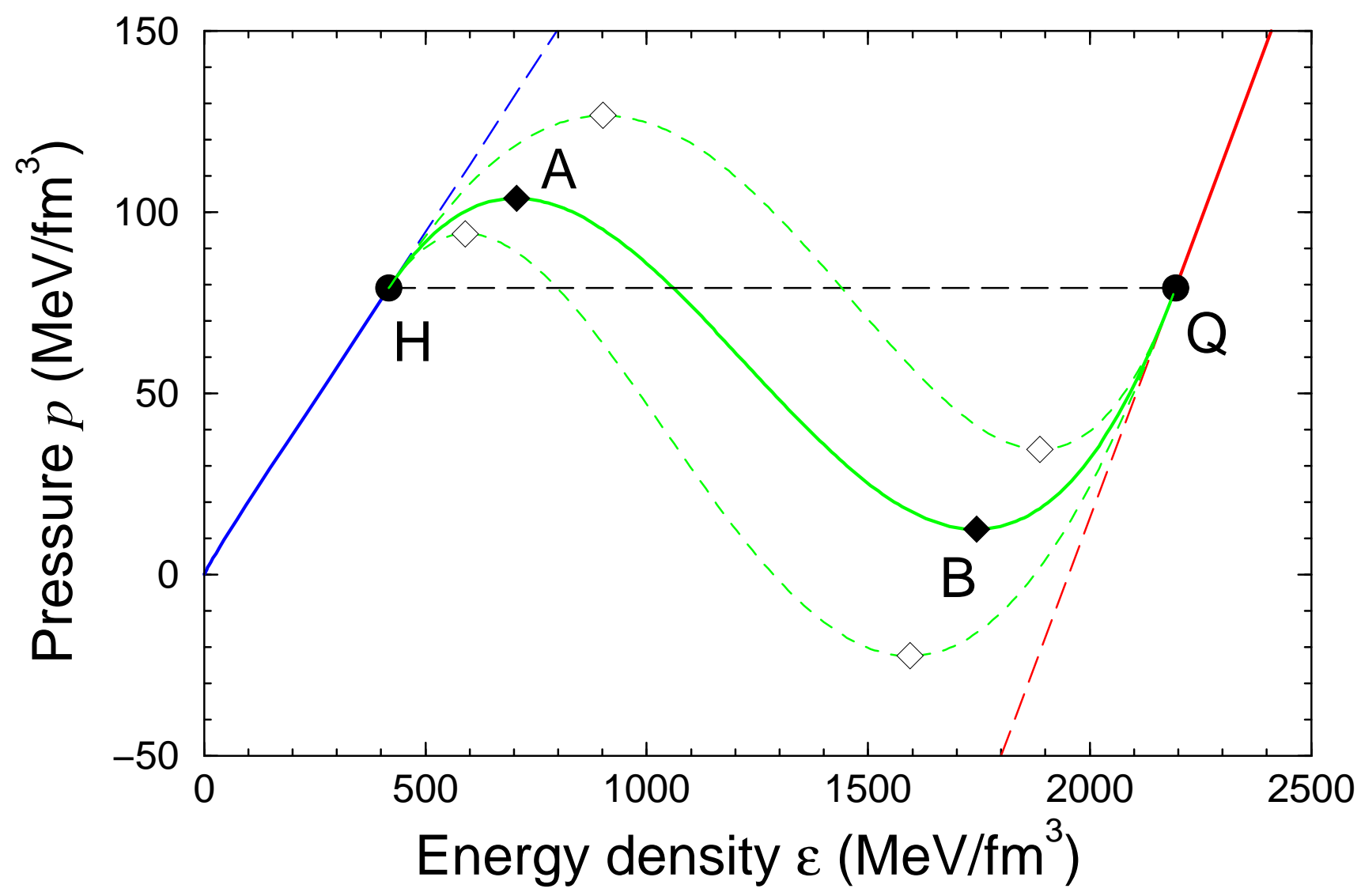

Figure 1 


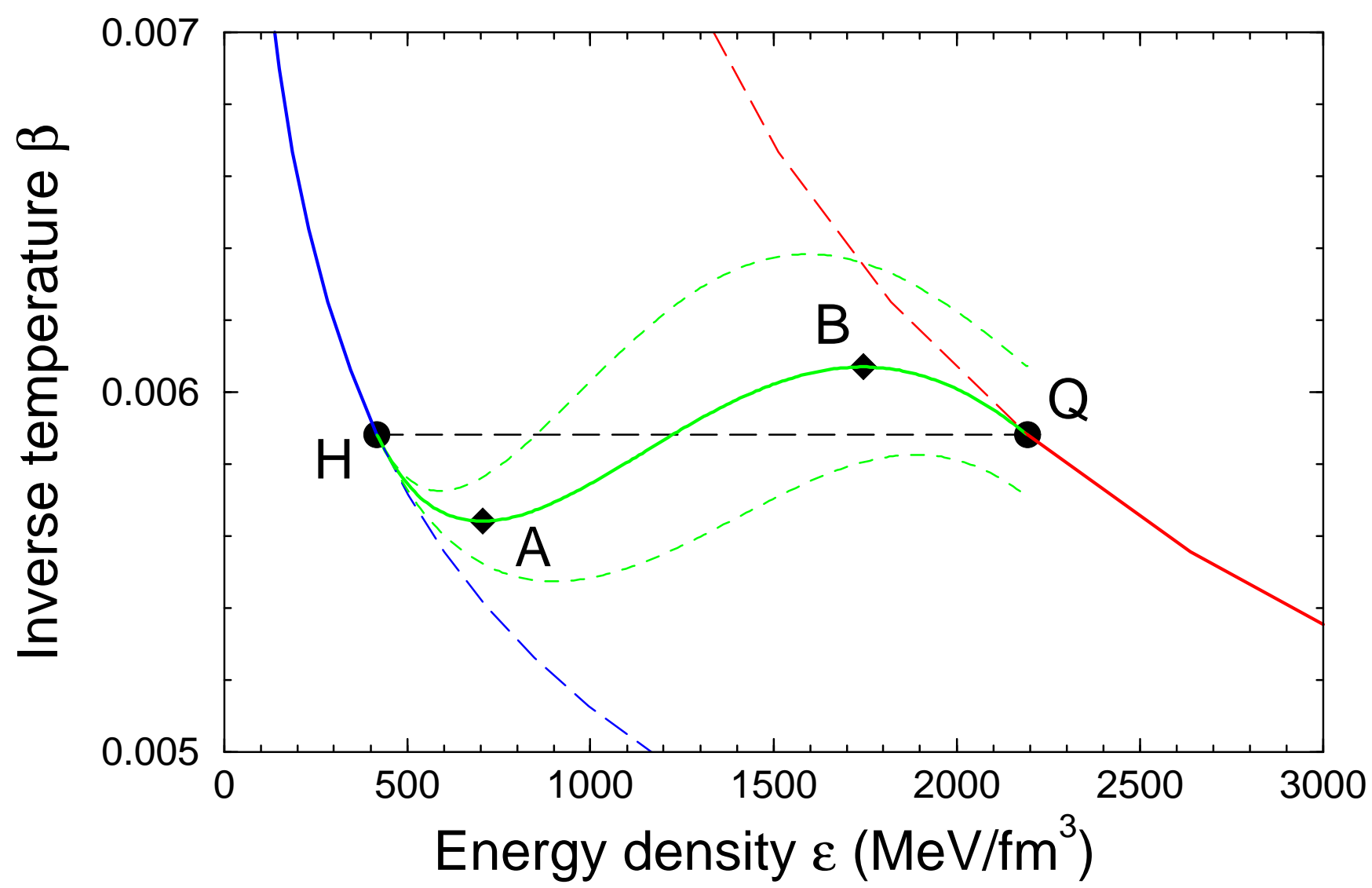

Figure 2 


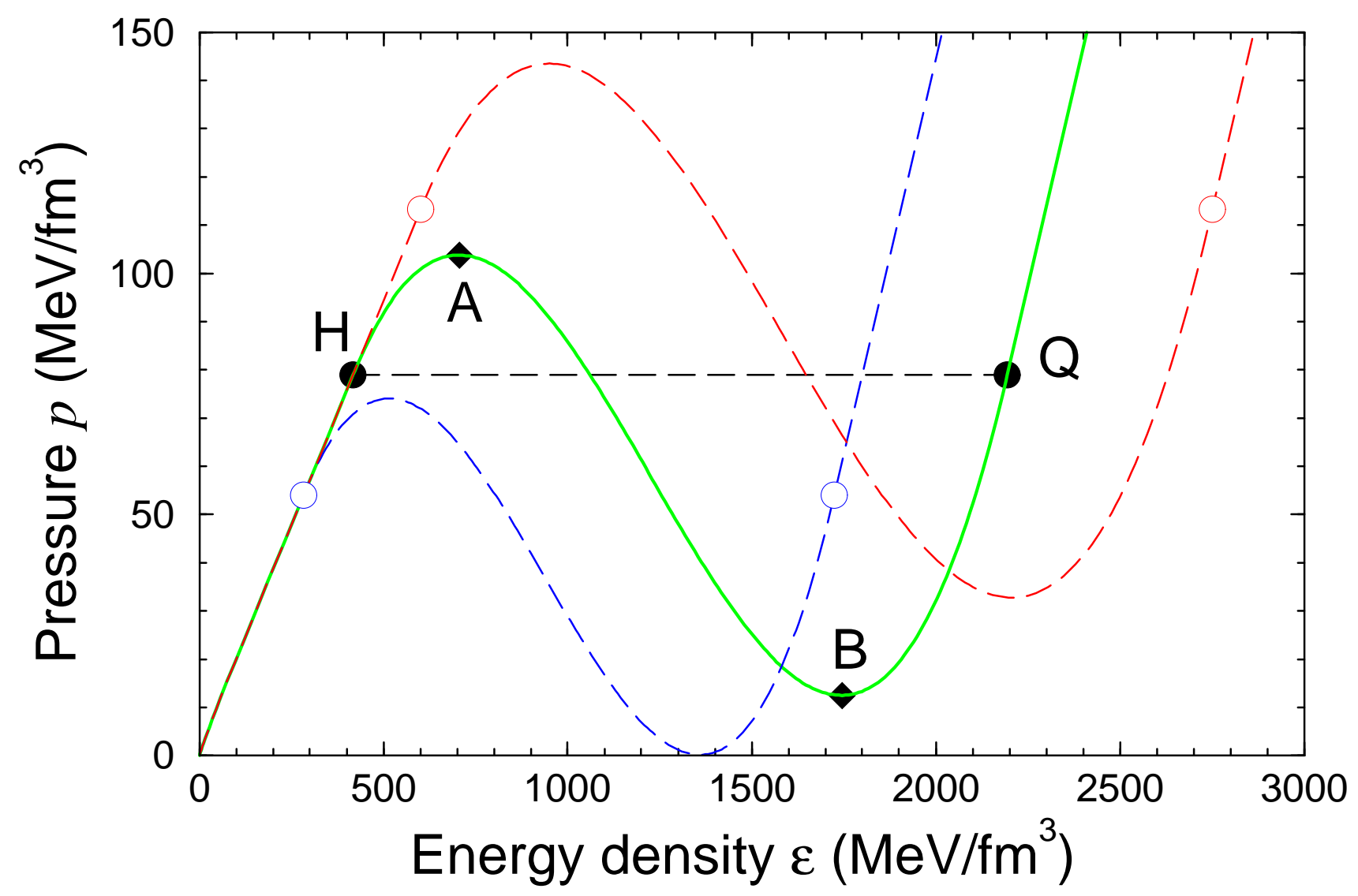

Figure 3 


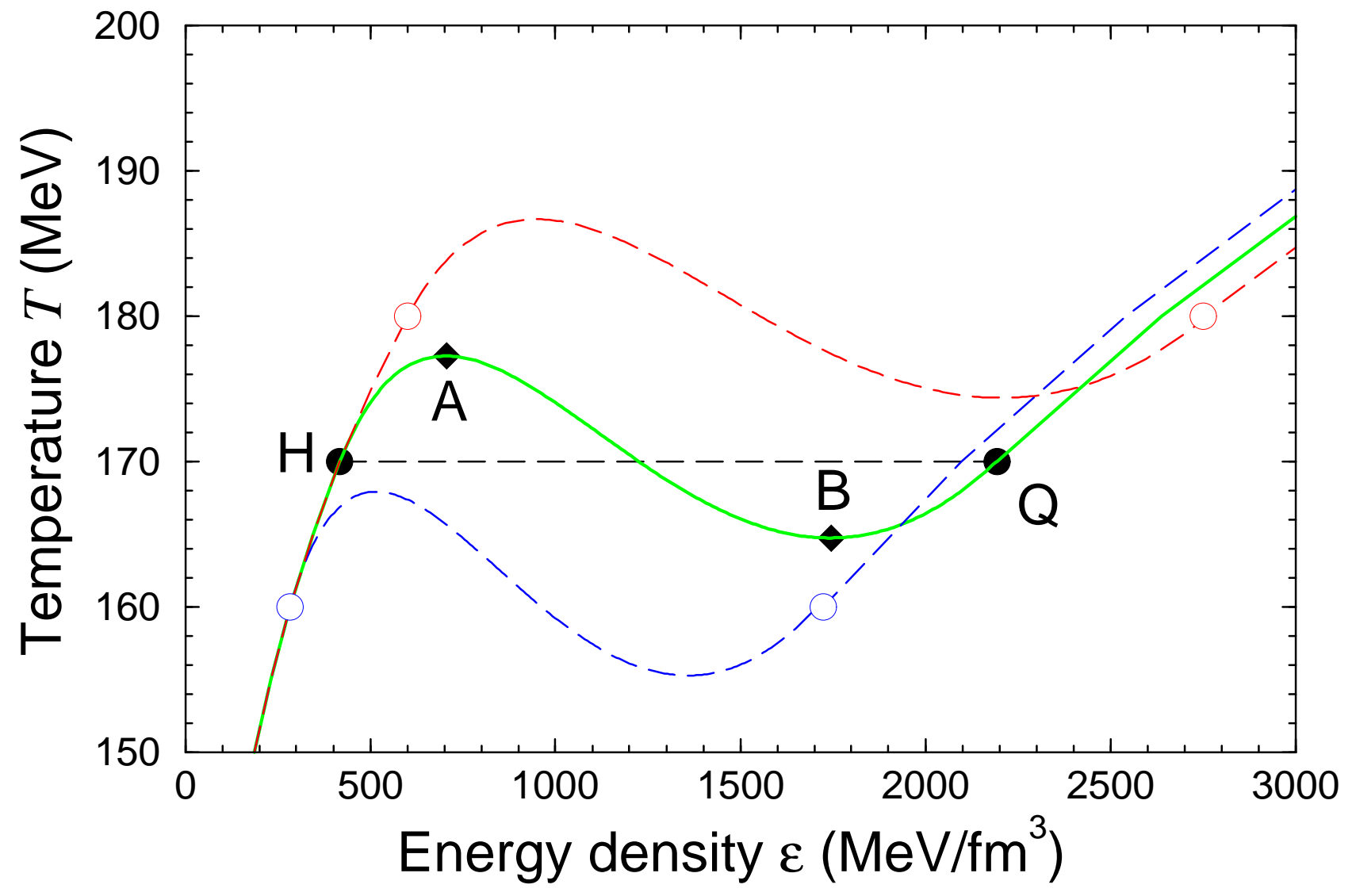

Figure 4 